\title{
La logística preventiva y su incidencia en la competitividad de la empresa
}

\author{
Lic. Víctor ACOSTA BUENO
}

\section{RESUMEN}

La logística es el campo de la gestión empresarial que se encarga directa e indirectamente, de sostener un apoyo oportuno, preciso y económico a las actividades administrativas y operativas de la empresa, para que estas no vean interrumpidas sus operaciones internas y externas, que son en si mismas la razón de ser de la organización.

La logística no es solo una actividad, son un conjunto de técnicas y procesos que actuando de forma sistemática y metódica, logran brindar un soporte sostenido y flexible ante la diversidad de requerimientos de las áreas administrativas y operativas de una empresa u organización, sean estas organizaciones de orden privado o público.

En la actividad empresarial privada se pierden millones de nuevos soles al año como consecuencia de las paralizaciones de procesos y servicios, que se producen por la causal de "fallas e imprevistos" que en realidad no son otra cosa, que la negligencia del administrador del proceso o servicio para detectar a tiempo oportuno la necesidad del mantenimiento preventivo que evite esa paralización.

En el sector público la falta de una cultura de logística preventiva trae como consecuencia, la ostensible reducción del nivel de servicio al público en general y con ello el incremento de reclamos e incomodidad del mismo.

Las empresas privadas indiscutiblemente buscan entre otros objetivos estratégicos, la satisfacción plena del cliente y la rentabilidad, ya que el primero es impulsor del segundo. Pero las empresas u organizaciones del sector estatal o público buscan entre otros objetivos estratégicos: alcanzar un óptimo "grado de alistamiento logístico" de sus unidades operativas que brindan servicios al público en general. 


\title{
PALABRAS CLAVES:
}

Grado de alistamiento: Nivel de preparación operativo y administrativo para enfrentar con éxito requerimientos y exigencias de diversas magnitudes.

\begin{abstract}
Logistics is the field of business management that is responsible directly and indirectly, to hold a timely, accurate and economic support to the administrative and operational activities of the company, so that they see not interrupted its internal and external operations, which are themselves the reason for the organization. Logistics is not only an activity, are a set of techniques and processes acting in a systematic and methodical way, manage to provide a sustained and flexible support to the diverse requirements of the administrative and operational areas of a company or organization, are these organizations Private or public order. In private business activity million soles annually are lost due to stoppages of processes and services that occur on the grounds of "unforeseen failures" that are really nothing, that the negligence of the process administrator or service to detect the need for timely preventive maintenance is to avoid paralysis. In the public sector the lack of a culture of preventive logistics results in the obvious reduction in the level of service to the general public and thereby increasing complaints and discomfort of it. Private companies seeking unquestionably among other strategic objectives, customer satisfaction and profitability, since the former is the second impeller.
\end{abstract}

But companies or organizations seeking state or public sector including strategic objectives: achieving optimal "degree of logistical readiness" of its operating units that provide services to the general public.

¿Qué es el grado de alistamiento logístico?.- Entendemos por "Grado de alistamiento logístico" a la capacidad de respuesta inmediata y eficiente de una organización logística ante diversos tipos de demandas, sean estas de carácter rutinario o de emergencia, procedentes del interior o exterior de la empresa.

Sobre todo, se pone a prueba el grado de alistamiento logístico en el caso de requerimientos que revisten el carácter de urgencia o emergencia.

La idea fundamental de esta teoría; Logística Preventiva, no es otra que la de dejar claramente definida la indiscutible necesidad de aplicar la técnica del mantenimiento preventivo a todos los equipos de las plantas de operaciones y a los equipos de los procesos administrativos, de tal manera que ninguno de ellos falle, se paralice o disminuya su rendimiento por: desgaste de material, tiempo de uso, ciclos cumplidos, disminución en su capacidad productiva o rendimiento, imprevistos. 
Esta técnica de mantenimiento continuo, dentro de la Logística Preventiva, es aplicable a todo tipo de empresas, tanto del rubro comercial, industrial, o de servicios. Por ejemplo; una tienda de auto servicio con un abastecimiento continuo de los productos en los estantes para evitar malestar del cliente, una industria manufacturera que requiere mantener una producción continua para evitar que por falta de abastecimiento vea desperdiciada su capacidad instalada, una entidad prestadora de servicios de salud como podría ser una clínica o un hospital, sobre todo y en forma muy especial en este último caso, donde la gestión del mantenimiento de equipos biomédicos adquiere relevancia en la administración hospitalaria, debido a que las instituciones prestadoras de servicios de salud (IPSS), requieren de una logística interna estructurada, que vaya de la mano con la gestión del mantenimiento de estos, lo que permite prestar los servicios asistenciales oportunamente, al predecir y prevenir los daños emergentes de los mismos.

Las fallas en los equipos biomédicos por falta de un mantenimiento preventivo, es decir de una Logística Preventiva, traen como consecuencia, la interrupción en el normal funcionamiento de los procesos asistenciales, así como de los procedimientos médicos, afectando la calidad y oportunidad de la prestación del servicio de salud al usuario y su percepción de calidad del mismo. El proceso de mantenimiento en sí mismo, tanto para las empresas cuyas operaciones son críticas y de alta incidencia en el usuario, sean estos cliente internos o externos, se compone de diversas acciones que tienden a minimizar o eliminar totalmente las fallas o deficiencias en los equipos para que estos puedan brindar un rendimiento óptimo y continuo, tanto en cantidad de servicios, productos elaborados, así como en calidad de los mismos.

El mantenimiento aplicado en la logística preventiva, para equipos que están sometidos a intensa actividad industrial o de servicios, se compone de ciertos elementos a tener en cuenta:

1. Planificación del mantenimiento en el corto plazo (entre uno y tres meses), mediano plazo (entre tres y seis meses) y en el largo plazo (entre seis meses y un año o más).- Tomar en cuenta la recomendación del fabricante o empresa matriz comercial de los equipos o maquinaria.

2. Seguimiento y control del funcionamiento por parte del usuario y del área logística.- Este control debe efectuarse en base a parámetros de medición, que generalmente vienen dados por el fabricante y que van disminuyendo en relación directa al tiempo de uso y vida útil del equipo. También es factible aplicar parámetros de control en base a promedios de rendimiento obtenidos por estadísticas o historia de funcionamiento.

3. Contrastación de incidencias o fallas en los equipos, antes y después del mantenimiento logístico preventivo, siempre en relación a parámetros 
estandarizados de rendimiento óptimo en condiciones normales de exigencia de cada equipo.

4. Identificación de puntos álgidos o débiles en los procesos de operación críticos que deben ser sometidos a mantenimiento preventivo intenso o rutinario.

5. Rediseño del programa de mantenimiento logístico preventivo, en base a los resultados obtenidos de la medición o contrastación anterior.

6. Identificar oportunidades y fortalezas con personal propio de mantenimiento, generalmente para equipos que no revisten alta criticidad en su operación, así como identificar oportunidades y fortalezas de las fuentes de abastecimiento y proveedores de servicios, que por lo general deberá ser proveedores especializados de equipos que revisten alta criticidad.

7. Aplicar criterios de asignación de prioridades de atención a las solicitudes de mantenimiento preventivo de equipos, sobre todo cuando se presentan en simultáneo, para así garantizar la atención prioritaria a aquellos equipos que representan o sustentan las operaciones de mayor criticidad y riesgo.

8. Aplicar indicadores de gestión para el control del mantenimiento preventivo en función a: Reclamos de usuarios, paralizaciones de equipos.

Si aplicamos una política de Logística Preventiva con mantenimientos programados y priorizados, definitivamente se logrará un impacto positivo en la rentabilidad de la empresa, con un significativa reducción de costos en las operaciones (entre un $15 \%$ a un $45 \%$ ), esto es principalmente porque debido al mantenimiento oportuno de los equipos de planta y administrativos, se mejorará su rendimiento y productividad, acortando tiempos y disminuyendo el número de fallas o paralizaciones por imprevistos.

Por otra parte, se obtendría mayor satisfacción del usuario externo disminuyendo significativamente el índice de reclamos.

Para que la logística preventiva tenga éxito y sea realmente eficaz en el objetivo estratégico de disminuir o eliminar las paralizaciones de los equipos, procesos o sistemas, es condición Sine Qua Non, que el administrador del equipo, proceso o sistema deba estar convencido y comprometido con la cultura de la prevención, ser consciente que sin su decidido apoyo no se le logrará el objetivo esperado. Después de todo la Logística no es una actividad con una incidencia solo vertical sino principalmente transversal.

La logística preventiva es más que una técnica de abastecimientos oportunos, es una corriente de pensamiento que permite concientizar, enraizar la cultura de la prevención en los usuarios y viabilizar la cadena de suministros, siendo a 
su vez un factor diferenciador de competitividad en el mercado para la empresa que lo aplica.

$\mathrm{Su}$ aplicación facilita la mejora de la productividad por el aprovechamiento al máximo de las horas de trabajo de los equipos y procesos, el fortalecimiento de la competitividad e imagen de la empresa ante sus clientes.

\section{BIBLIOGRAFÍA REFERENCIAL}

1. Ballou, Ronald Logística. Administración de la Cadena de Suministro, Edición Pearson Prentice Hall, México 2005

2. Gutierrez Casas, Gil Logística y Distribución Física, McGraw Hill Interamerica de España, 2008

3. Tawfik, L. y Chauvel, M. Administración de la Producción, Interamericana S.A México, 2010

4. Acosta Bueno, Víctor Logística Empresarial Moderna, Talleres Gráficos Artes e impresiones, Lima, 2004. 


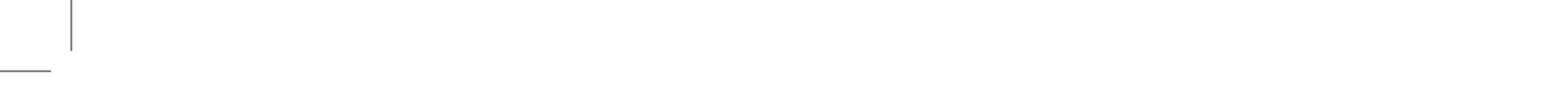

